\title{
Differentiating fallers from nonfallers using nonlinear variability analyses of data from a low-cost portable footswitch device: a feasibility study
}

\author{
Arash Mohammadzadeh Gonabadi ${ }^{1,2 *}$, Prokopios Antonellis ${ }^{1}$, PhilipPe MAlCOLM $^{1}$ \\ ${ }^{1}$ Department of Biomechanics and Center for Research in Human Movement Variability, \\ University of Nebraska at Omaha, Omaha, Nebraska, United States of America. \\ ${ }^{2}$ Rehabilitation Engineering Center, Institute for Rehabilitation Science and Engineering, \\ Madonna Rehabilitation Hospitals, Lincoln, Nebraska, United States of America.
}

\begin{abstract}
Purpose: Falls are one of the main causes of injuries in older adults. This study evaluated a low-cost footswitch device that was designed to measure gait variability and investigates whether there are any relationships between variability metrics and clinical balance tests for individuals with a history of previous falls. Methods: Sixteen older adults completed a history of falls questionnaire, three functional tests related to fall risk, and walked on a treadmill with the footswitch device. We extracted the stride times from the device and applied two nonlinear variability analyses: coefficient of variation and detrended fluctuation analysis. Results: The temporal variables and variability metrics from the footswitch device correlated with gold-standard measurements based on ground reaction force data. One variability metric (detrended fluctuation analysis) showed a significant relationship with the presence of past falls with a sensitivity of $43 \%$. Conclusion: This feasibility study demonstrates the basis for using low-cost footswitch devices to predict fall risk.
\end{abstract}

Key words: aging, balance, falls, nonlinear dynamics, variability, walking

\section{Introduction}

Falls are an important risk for our aging population and are associated with high economic costs [7]. About one out of three adults older than 65 falls per year [15]. A variety of possible contributing factors include visual impairment and cognitive decline [8]. One study showed the relationship between gait mechanics and future falls [18]. Gait is a complex cyclic behavior characterized by slight variations between strides.

It has been theorized that a healthy state is associated with an optimal level of variability, which allows the system to adapt to varying circumstances [25]. A pathological state can be either too regular or too random. Moreover, studies have demonstrated a prospective relationship between gait variability and falls [12], [18], [23], [28]. Gait variability of biomechanical measures (joint kinetics and kinematics, stance and swing times, stride widths and lengths, minimum foot clearances) can differentiate fall-prone and healthy older adults [1], [10], [21], [28]. Additionally, older adult fallers exhibit increased gait variability [14], [15]. Associations between falls and gait variability are variable and task-dependent [3], [24].

Most gait analysis systems are costly and laboratory-based. The rationale for developing low-cost sys-

\footnotetext{
* Corresponding author: Arash Mohammadzadeh Gonabadi, Department of Biomechanics and Center for Research in Human Movement Variability, University of Nebraska at Omaha, 6160 University Drive South, Omaha, NE 68182, United States of America. E-mail: amgonabadi@unomaha.edu

Received: December 18th, 2020

Accepted for publication: April 16th, 2021
} 
tems that enable portable monitoring of gait variability is that these systems could allow for early detection of changes in fall risk. Our aim was to develop a lowcost portable device that could be used to measure gait variability. Finally, we conducted a preliminary evaluation to investigate if measurements with this device could differentiate individuals who previously experienced a fall from nonfallers in a cross-sectional feasibility study.

\section{Materials and methods}

\subsection{Participants}

Sixteen healthy older adults ( 7 males, 9 females; $71 \pm 6$ years; $74 \pm 16 \mathrm{~kg} ; 1.71 \pm 0.11 \mathrm{~m})$ participated in this study (Table 1). The sample size was based on similar feasibility studies [26], [27] and guidelines on adequate sample sizes for pilot studies [16]. Participants provided informed consent, and the study was approved by the University of Nebraska Medical Center Institutional Review Board. All participants were able to walk independently without an assistive device, did not suffer from neurological disease and any lower limb disabilities, injuries, or disease. Older adult participants have experienced one fall within the past year that was determined through self-reporting. The exclusion criteria for both young and older adult participants were any neurologic conditions or lower limb disabilities or disease that could affect gait and unable to walk unassisted.

Table 1. Participant characteristics

\begin{tabular}{|c|c|c|c|c|}
\hline Participant & Gender & Age [years] & Height $[\mathrm{m}]$ & Body mass $[\mathrm{kg}]$ \\
\hline 1 & Female & 70 & 1.55 & 60.78 \\
\hline 2 & Male & 77 & 1.75 & 63.63 \\
\hline 3 & Female & 71 & 1.58 & 62.73 \\
\hline 4 & Male & 72 & 1.83 & 85.45 \\
\hline 5 & Female & 68 & 1.64 & 67.27 \\
\hline 6 & Female & 70 & 1.63 & 59.09 \\
\hline 7 & Male & 65 & 1.91 & 88.18 \\
\hline 8 & Male & 71 & 1.88 & 104.55 \\
\hline 9 & Male & 60 & 1.80 & 111.36 \\
\hline 10 & Female & 70 & 1.70 & 77.56 \\
\hline 11 & Female & 76 & 1.60 & 57.15 \\
\hline 12 & Female & 83 & 1.65 & 67.13 \\
\hline 13 & Female & 79 & 1.78 & 76.20 \\
\hline 14 & Male & 76 & 1.65 & 77.11 \\
\hline 15 & Female & 66 & 1.70 & 61.24 \\
\hline 16 & Male & 67 & 1.65 & 70.30 \\
\hline
\end{tabular}

\subsection{Experimental procedure}

We examined whether there was a relationship between gait variability parameters and the presence of falls in a cross-sectional study design. Participants reported if they had experienced a fall (defined as "a slip or trip that resulted in a loss of balance and landing on a lower level") in the past year. Attitudes toward falls were assessed using the Modified Falls Efficacy Scale questionnaire. This is a questionnaire that measures the fear of falling in older adults. Participants also filled out the EQ-5D-5L health questionnaire. This is another scale in which the participants should rate their health on a vertical visual analogue scale. We assessed balance and gait with the Timed Up and Go test, the Modified Dynamic Gait Index (MDGI), and the Berg Balance Scale. Timed Up and Go test evaluates balance, walking performance, and fall risk. The MDGI was designed to evaluate the dynamic balance during walking. The Berg Balance Scale was designed to assess the balance performance and determine the fall risk in an older population. We measured stride and stance times during ten minutes of walking at $0.8 \mathrm{~m} \cdot \mathrm{s}^{-1}$ on a force treadmill (1000 Hz; Bertec, Columbus, OH).

\subsection{Footswitch system}

We developed a footswitch system that consisted of pressure sensors (FlexiForce, South Boston, MA) and a microcontroller $(250 \mathrm{~Hz}$; Adafruit, New York City, NY) (Fig 1). The pressure sensors are trimmable and include a 3-pin male connector. The force range

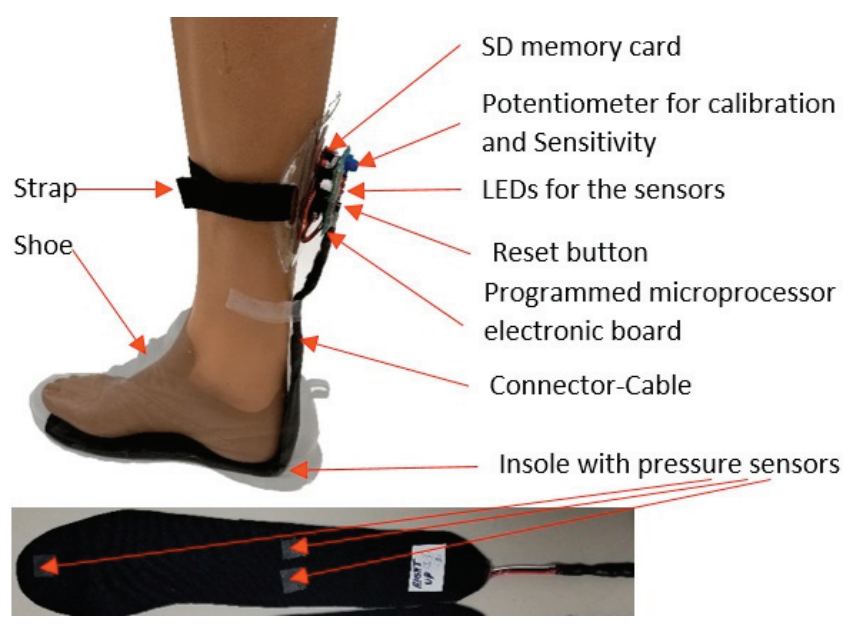

Fig. 1. Low-cost footswitch device. The picture shows the insole with embedded pressure sensors and electronics. A transparent shoe is portrayed to show how the insole fits inside a shoe 
of the sensors is $445 \mathrm{~N}(0-100 \mathrm{lb})$. The dynamic range of this sensor, versatile force sensor can be modified by changing the drive voltage and adjusting the resistance of the feedback resistor. We selected this microcontroller because it is easy to use in portable devices as well as including a USB to serial program and debug capability besides, the heart is clocked at $8 \mathrm{MHz}$ and at $3.3 \mathrm{~V}$ logic. This chip has $32 \mathrm{~K}$ of flash and $2 \mathrm{~K}$ of RAM. To compare measurements against a gold standard, we also measured vertical ground reaction forces (GRF) and detected gait events with the force treadmill.

\subsection{Data processing}

In the GRF data, heel strikes and toe-offs were detected when the unfiltered vertical GRF crossed 5\% of the participant's body weight. The footswitch data were filtered with a $10 \mathrm{~Hz}$ Butterworth filter. Heel strikes were detected using a threshold of $15 \%$ of the range between the minimum and maximum force signal. Toe-offs were detected using a threshold of $30 \%$. We removed errors in the gait event detection (e.g., due to crossing the split between the treadmill force plates or due to pressure from the foot on the insole during the swing phase) by plotting the stride normalized GRF and footswitch signals and excluding strides that fell outside of a band of the mean (plus or minus 2.5 times the interquartile range).

We calculated two gait variability metrics from the recorded stride times and stance times: the coefficient of variation $(100 \times$ standard deviation/mean $)$ [2], [11], [14] and the detrended fluctuation analysis (DFA) scaling coefficient [9]. We used data obtained for both legs, except in 5 out of the 16 participants in which one of the footswitches malfunctioned. Moreover, we calculated sensitivity and specificity. Sensitivity is defined as the proportion of participants who were correctly identified as fallers divided by the number of participants who were correctly detected as fallers plus the participants who were incorrectly detected as nonfallers. Specificity is defined as the proportion of participants who were correctly identified as nonfallers divided by the number of participants who were correctly identified as nonfallers plus the number of participants who were incorrectly defined as fallers.

\subsection{Statistical analyses}

We compared temporal variables and variability metrics versus the GRF-based measurements (gold stan- dard) using Pearson's correlation. We analyzed the relationships between the gait variability metrics and fall history using logistic regression and the relationships between the gait variability metrics and the balance questionnaires and tests using linear regression. All analyses were performed in MATLAB (MathWorks, Natick, MA).

\section{Results}

We found a strong correlation between the stride time measured with the footswitch device and the stride time based on the GRF measurements $(R=$ $100 \%, P<0.001)$ as well as moderate to strong correlations between footswitch-based and GRF-based variability metrics $\left(R_{\text {coefficient of variation }}=0.98, P<0.01\right.$; $\left.R_{\mathrm{DFA} \text { scaling coefficient }}=0.58, P=0.001\right)$. We found a weak but significant relationship between the stance time DFA scaling coefficient measured with the footswitch device and the reporting of previous falls ( $P$-value 0.024 , logistic regression; Fig. 2). We did not find any other significant relationships between any of the variability metrics and the questionnaire results and functional balance tests ( $P$-values $\geq 0.063$, linear regression; Figs. 3, 4).

Only footswitch-based stance time DFA scaling exponent shows a significant relationship with reported falls. Sensitivity (i.e., the number of true positives divided by the number of positives) is slightly larger than specificity (i.e., the number of true negatives divided by the number of negatives). This means that the footswitch-based stance time DFA scaling exponent is better at estimating who did not report a fall than at estimating who reported a fall. The measurement sensitivity of the footswitch system was determined by the FlexiForce sensors attached to the insoles. According to the datasheet of the sensors, collecting data utilizing an Op Amp Circuit (operational amplifier) [5], the linearity (error) was less than $\pm 3 \%$, the repeatability was less than $\pm 2.5 \%$, the hysteresis was less than $\pm 4.5 \%$, and the response time was less than 5 .

\section{Discussion}

This study examined a low-cost, easy-to-implement footswitch system designed to measure gait variability and investigated if there are relationships between gait variability metrics and clinical balance tests for fall 

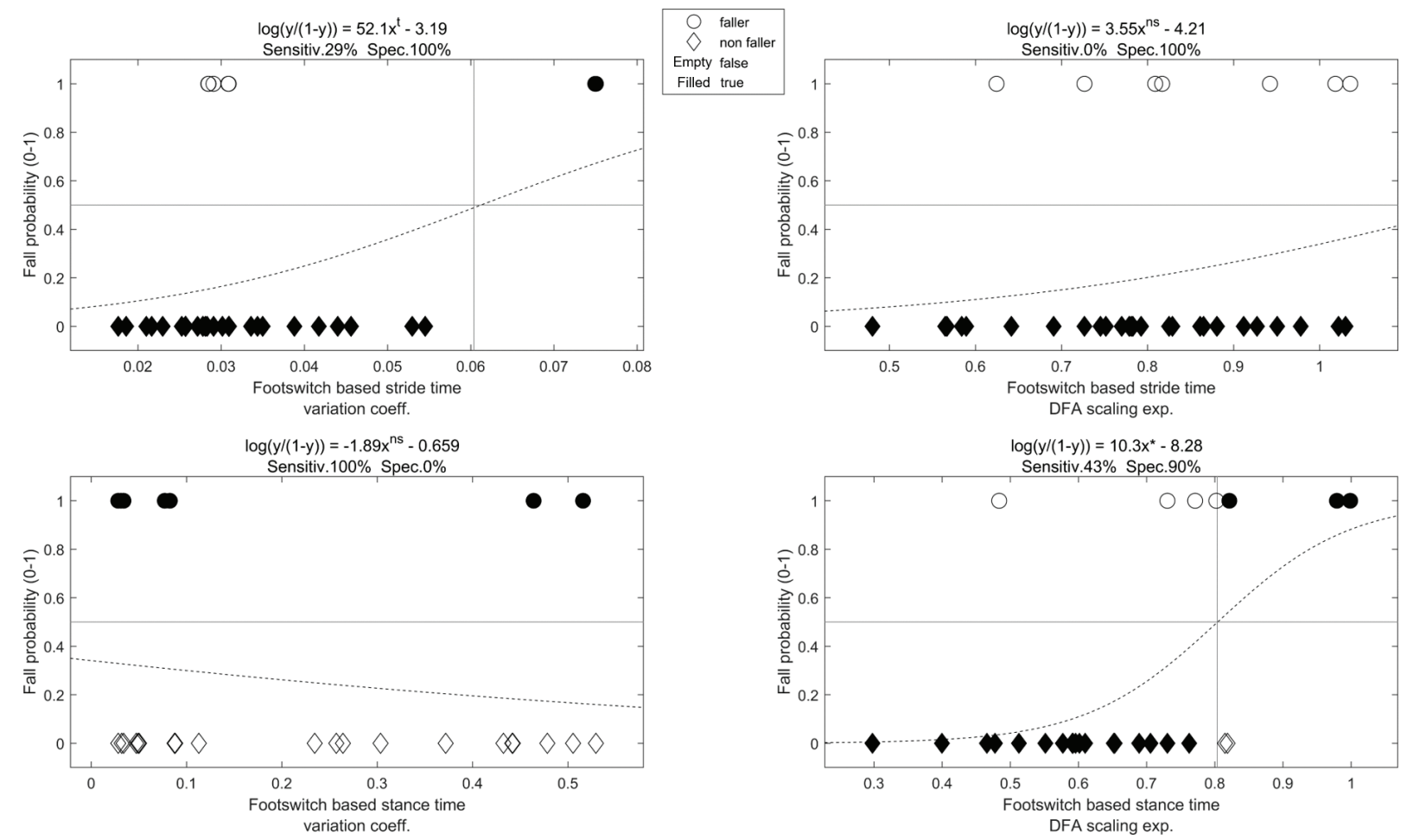

Fig. 2. Reported falls versus variability measurements with the footswitch device. Reported falls are shown on vertical axes

( 1 indicates a reported fall, 0 indicates no reported fall). Variability measurements are shown on the horizontal axes.

The dashed line represents a curve fit with logistic regression. The inset text shows the equation of the logistic regression curve fit as well as sensitivity and specificity. Trials categorized correctly according to the logistic regression equation are marked with a cross.

Trials identified incorrectly are marked with a diamond (filled diamonds $=$ true negatives, filled circles $=$ true positives, empty circles = false negatives, empty diamonds = false positives; negative means detected as nonfaller; positive means detected as faller).

Significance of the logistic regression is indicated with symbols. * is $P \leq 0.05, t$ is $P \leq 0.10, n s$ is $P>0.10$
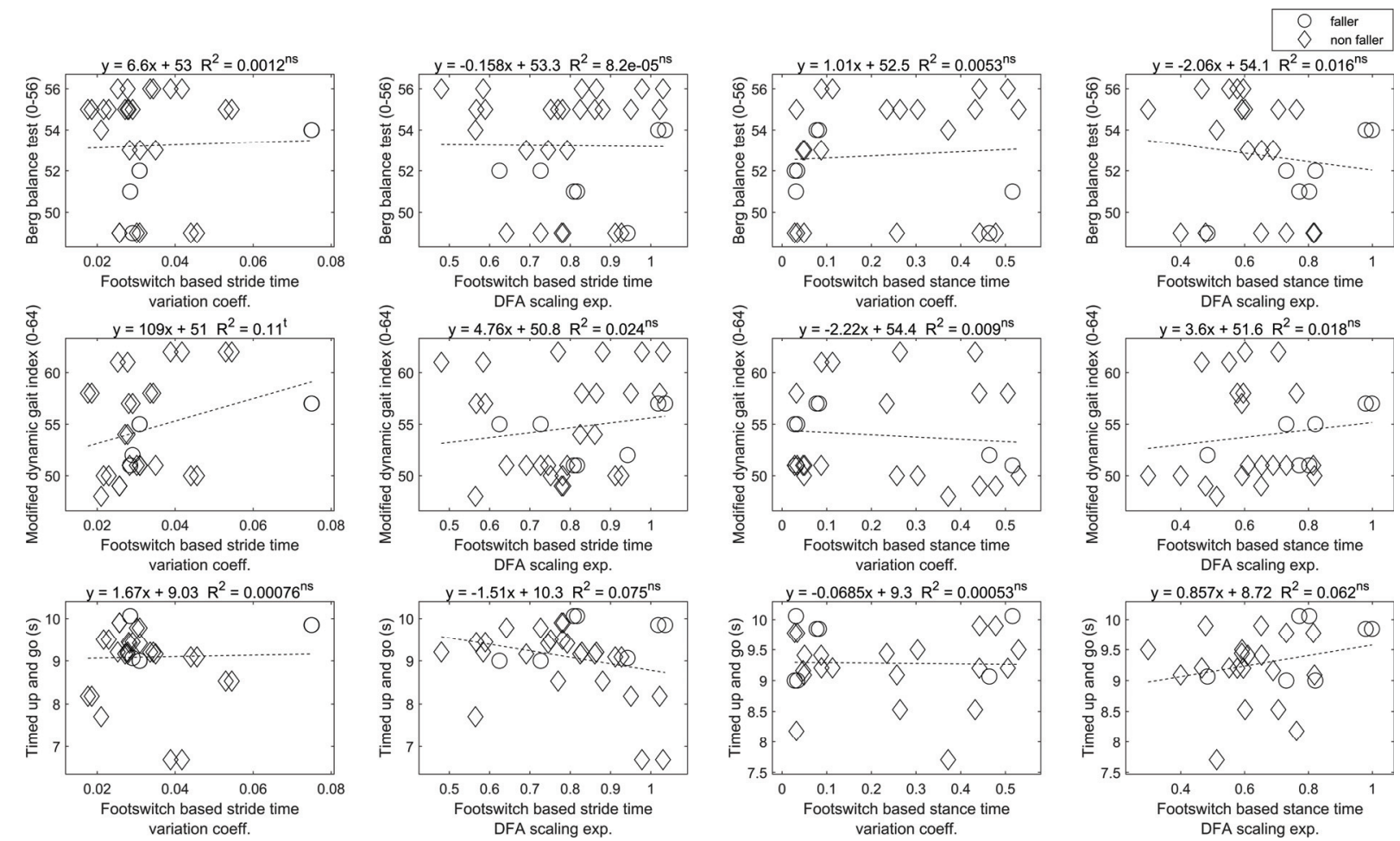

Fig. 3. Balance tests versus variability measurements with the footswitch device. Balance tests are shown on the vertical axes. Footswitch-device based variability coefficients are shown on the horizontal axes. Lines indicate regression lines.

The inset text shows the regression formula and the coefficient of determination. $t$ is $P \leq 0.10, n s$ is $P>0.10$ 

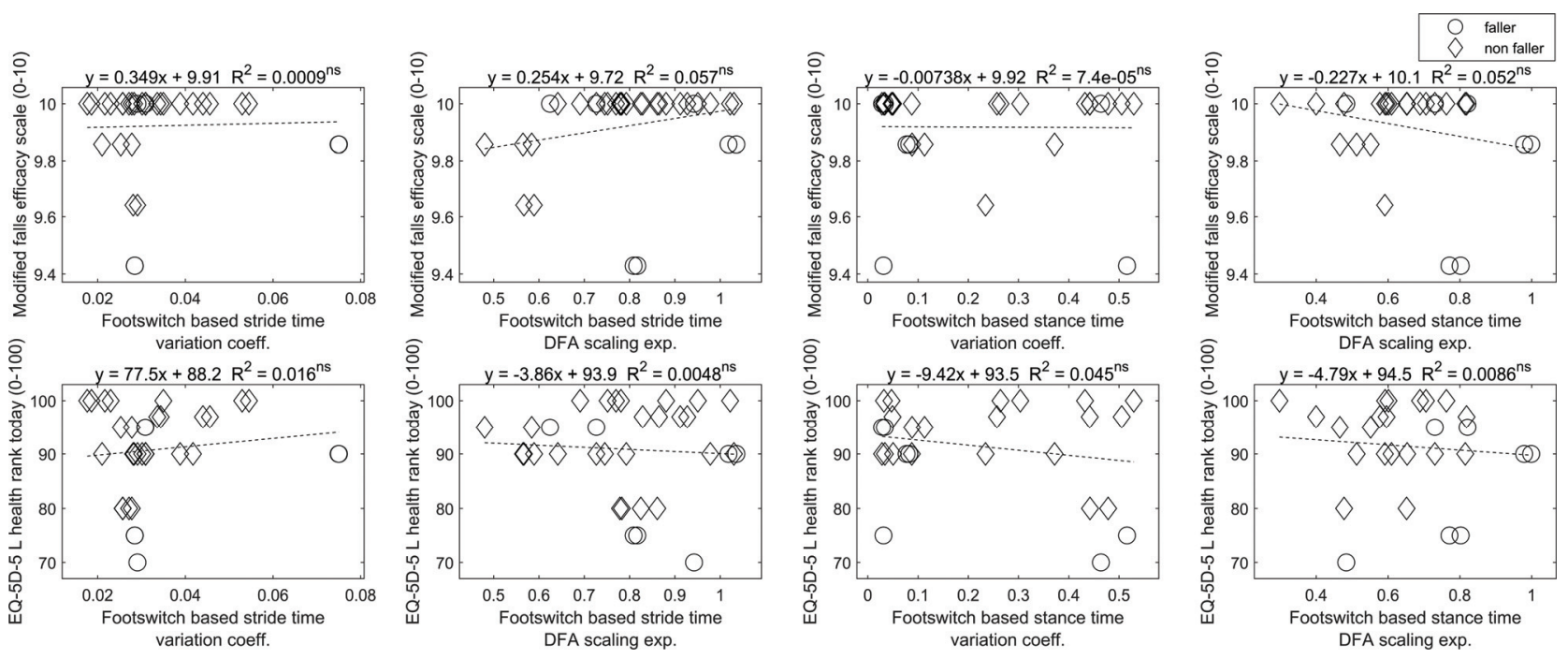

Fig. 4. Balance and health questionnaires versus variability measurements with the footswitch device.

Questionnaires are shown on the vertical axes. Footswitch-device based variability coefficients are shown on the horizontal axes.

Lines indicate regression lines. The inset text shows the regression formula and the coefficient of determination. $n s$ is $P>0.10$

history in older adults. We found that stance time DFA measured with the footswitch device differentiates previous fallers with a sensitivity of $43 \%$ and specificity of $90 \%$ (Fig. 2). While the sensitivity is not high, this is a similar magnitude for sensitivity and specificity to that achieved in certain studies with functional tests in other populations (e.g., sensitivity of $35-57 \%$ and specificity of $87-100 \%$ ) [6].

According to the results of this study, fallers had a longer double-limb stance time, consistent with other research [4]. A reason for this could be the loss of muscle strength and joint flexibility due to aging [17]. Potentially, this difference in stance time duration could be related to the difference in stance time DFA scaling coefficient values between the two groups in our study. Other studies also found changes in similar coefficients that explain a more random gait pattern in older adults [9], similar to the difference in DFA scaling coefficients between the two groups in our study.

We found moderate to strong correlations between temporal and gait variability metrics measured with the footswitch device and the GRF-based results, which indicate that the footswitch device is sufficiently advanced to measure gait variability in a portable manner. Previously, it was shown that foot contact events identified with footswitches agree adequately with GRF-based methods [20].

There were no significant relationships between the balance and gait tests and the measurements from our device. This could be related to the subjective nature of some tests. There was only a weak trend toward a significant relation between the stride time coefficient of variation and the modified dynamic gait index (Fig 3). The need for self-control, independence in activities, and knowledge of basic safety features are important motivations to use new technologies to determine the risk of falls in older adults [13]. Clinical balance assessment tools may sometimes not be accurate enough to distinguish the balance deficits associated with gait [19], which indicates the importance of using technology to assist with fall risk detection.

The current sample size and the fact that we did not recruit a representative average population of older adults limits the generalizability of the results. We also found only one significant metric that differentiates participants with previous falls from nonfallers, which could perhaps be a result of this. Another limitation is that this is a cross-sectional feasibility study. Investigating whether measurements with a similar device could predict falls prospectively could be a challenging but impactful next step.

Laboratory-based devices such as the force treadmill allow for more accurate and higher frame rate measurements. Future improvements to the footswitch device could include increasing the framerate to the same rate of force treadmills and evaluating if combinations of parameters (e.g., variability metrics and treadmill speed) can improve differentiating between fallers and nonfallers. Another possible improvement could involve adding extra pressure sensors to cover the entire foot to obtain more accurate measurements of heel strike and toe-off if the foot contacts the ground in different locations. Devices, including the one in this study, could be worn during daily life for conducting prospective studies [22]. Such studies 
could be descriptive (i.e., following a group of older adults during their daily life and recording occurrences of falls), or they could include subgroups with individualized intervention strategies (e.g., contacting older adults to start a balance training intervention as soon as their sensors indicate that they have an increased risk of falling).

\section{Conclusions}

This prototype could enable monitoring of gait variability outside of the laboratory on an outpatient basis. We found that a portable device measures temporal variability metrics well enough to distinguish fallers within the dataset.

\section{Acknowledgements}

The authors would like to thank Mr. Travis Vanderheyden for help with the fabrication of the device, Mr. Alec Harp for his help with data collection, and Dr. Joe Runge from UNeMed for project planning. We also thank all the participants for their time and effort for participating in the study.

\section{Funding}

This study was supported by J. Brasch. Co. LLC and the National Institutes of Health [P20GM109090].

\section{References}

[1] BaraK Y., WagenaAR R.C., Holt K.G., Gait Characteristics of Elderly People With a History of Falls: A Dynamic Approach, Phys. Ther., 2006, 86, 1501-1510.

[2] Bizovska L., Svoboda Z., Kubonova E., Vuillerme N., HiRJAKOVA Z., JANURA M., The differences between overground and treadmill walking in nonlinear, entropy-based and frequency variables derived from accelerometers in young and older women - preliminary report, Acta Bioeng. Biomech., 2018, 20, 93-100.

[3] Brach J.S., Berlin J.E., VanSwearingen J.M., Newman A.B., STUDENSKI S.A., Too much or too little step width variability is associated with a fall history in older persons who walk at or near normal gait speed, J. Neuroeng. Rehabil., 2005, 2, 21.

[4] CHIU S.-L., CHOU L.-S., Variability in inter-joint coordination during walking of elderly adults and its association with clinical balance measures, Clin. Biomech., 2013, 28, 454-458.

[5] Coughlin R.F., Driscoll F.F., Operational amplifiers and linear integrated circuits, Prentice Hall, Upper Saddle River, NJ 2001.

[6] Dibble L.E., Lange M., Predicting falls in individuals with Parkinson disease: a reconsideration of clinical balance measures, J. Neurol. Phys. Ther., 2006, 30, 60-7.
[7] Florence C.S., Bergen G., Atherly A., Burns E., Stevens J., DRAKE C., Medical Costs of Fatal and Nonfatal Falls in Older Adults, J. Am. Geriatr. Soc., 2018, 66, 693-698.

[8] HaRwood R.H., Visual problems and falls, Age Ageing, 2001, $30,13-18$.

[9] Hausdorff J.M., Mitchell S.L., Firtion R., Peng C.K., CudKowicz M.E., Wei J.Y., Goldberger A.L., Altered fractal dynamics of gait: reduced stride-interval correlations with aging and Huntington's disease, J. Appl. Physiol., 1997, 82, 262-269.

[10] HAUSDORFF J.M., Gait dynamics, fractals and falls: Finding meaning in the stride-to-stride fluctuations of human walking, Hum. Mov. Sci., 2007, 26, 555-589.

[11] HausdorfF J.M., EdelberG H.K., Mitchell S.L., GOLDBERGER A.L., WEI J.Y., Increased gait unsteadiness in community-dwelling elderly fallers, Arch. Phys. Med. Rehabil., 1997, 78, 278-83.

[12] HAusdorfF J.M., Rios D.A., Edelberg H.K., Gait variability and fall risk in community-living older adults: A 1-year prospective study, Arch. Phys. Med. Rehabil., 2017, 82, $1050-1056$.

[13] Hawley-Hague H., Boulton E., Hall A., Pfeiffer K., TODD C., Older adults' perceptions of technologies aimed at falls prevention, detection or monitoring: A systematic review, Int. J. Med. Inform., 2014, 83, 416-426.

[14] Herman T., Giladi N., Gruendlinger L., HausdorfF J.M., Six weeks of intensive treadmill training improves gait and quality of life in patients with Parkinson's disease: a pilot study, Arch. Phys. Med. Rehabil., 2007, 88, 1154-1158.

[15] Hornbrook M.C., STEvens V.J., WingField D.J., Hollis J.F., GreENLICK M.R., ORY M.G., Preventing falls among community-dwelling older persons: results from a randomized trial, Gerontologist, 1994, 34, 16-23.

[16] JuLious S.A., Sample size of 12 per group rule of thumb for a pilot study, Pharm. Stat., 2005, 4, 287-291.

[17] KANG H.G., Dingwell J.B., Separating the effects of age and walking speed on gait variability, Gait Posture, 2008, 27, 572-577.

[18] MAKI B.E., Gait changes in older adults: predictors of falls or indicators of fear, J. Am. Geriatr. Soc., 1997, 45, 313-320.

[19] MANCINI M., HoRAK F.B., The relevance of clinical balance assessment tools to differentiate balance deficits, Eur. J. Phys. Rehabil. Med., 2010, 46, 239-48.

[20] Mills P.M., Barrett R.S., Morrison S., Agreement between footswitch and ground reaction force techniques for identifying gait events: Inter-session repeatability and the effect of walking speed, Gait Posture, 2007, 26, 323-326.

[21] Newstead A.H., WALden G.J., GitTER A.J., Gait Variables Differentiating Fallers from Nonfallers, J. Geriatr. Phys. Ther., 2007, 30, 93-101.

[22] Patel S., Park H., Bonato P., Chan L., Rodgers M., A review of wearable sensors and systems with application in rehabilitation, J. Neuroeng. Rehabil., 2012, 9, 21.

[23] Paterson K., Hill K., LyTHGO N., Stride dynamics, gait variability and prospective falls risk in active community dwelling older women, Gait Posture, 2011, 33, 251-255.

[24] Roos P.E., Dingwell J.B., Influence of simulated neuromuscular noise on movement variability and fall risk in a $3 D d y$ namic walking model, J. Biomech., 2010, 43, 2929-2935.

[25] Stergiou N., Harbourne R.T., Cavanaugh J.T., Optimal movement variability: A new theoretical perspective for neurologic physical therapy, J. Neurol. Phys. Ther., 2006, 30, 120-129. 
[26] Toebes M.J.P., Hoozemans M.J.M., Mathiassen S.E., DEKKER J., VAN DIEËN J.H., Measurement strategy and statistical power in studies assessing gait stability and variability in older adults, Aging Clin. Exp. Res., 2016, 28, 257-265.

[27] Tran S.T., Thomas S., DiCesare C., Pfeiffer M., Sil S., Ting T.V., Williams S.E., Myer G.D., Kashikar-Zuck S.,
A pilot study of biomechanical assessment before and after an integrative training program for adolescents with juvenile fibromyalgia, Pediatr. Rheumatol., 2016, 14, 43.

[28] Verghese J., Holtzer R., Lipton R.B., Wang C., Quantitative Gait Markers and Incident Fall Risk in Older Adults, Journals Gerontol. Ser. A Biol. Sci. Med. Sci., 2009, 64A, 896-901. 